\title{
Preliminary Investigations of Elemental Content, Microporosity, and Specific Surface Area of Porous Rocks Using PIXE and X-ray Microtomography Techniques
}

\author{
J. Bielecki ${ }^{a, *}$, S. BożeK ${ }^{a, b}$, E. Dutkiewicz ${ }^{a}$, R. HAjduK ${ }^{a}$, J. JARzYnA ${ }^{c}$, J. LekKi $^{a}$, \\ T. PieprzycA ${ }^{a}$, Z. Stachura ${ }^{a}$, Z. Szklarz ${ }^{a}$ And W.M. KWiateK ${ }^{a}$ \\ ${ }^{a}$ The Henryk Niewodniczański Institute of Nuclear Physics, Polish Academy of Sciences \\ E. Radzikowskiego 152, 31-342 Kraków, Poland \\ ${ }^{b}$ Medical College, Department of Pharmacokinetics and Physical Pharmacy, The Jagiellonian University \\ Medyczna 9, 30-688 Kraków, Poland \\ ${ }^{c}$ Faculty of Geology, Geophysics and Environment Protection, AGH University of Science and Technology \\ al. A. Mickiewicza 30, 30-059 Kraków, Poland
}

\begin{abstract}
Determination of physical properties of porous geological materials is of great importance for oil industry. The knowledge of rocks properties is usually obtained from porosity studies such as pore size distribution, specific surface area determination, and hydrodynamic permeability calculations. This study describes determination of elemental composition and measurements of the particular physical properties of geological samples (porous sandstone rocks) by means of the nuclear and X-ray microprobes at the Institute of Nuclear Physics, Polish Academy of Sciences in Kraków, Poland. The special emphasis has been put on the computed microtomography method. Measurements have been carried out in close cooperation with Department of Geophysics, FGGEP AGH in Kraków, Poland. Chemical composition of the Rotliegend sandstone rock samples (few millimeters diameter), extracted from a borehole at $2679.6 \mathrm{~m}, 2741.4 \mathrm{~m}$ and $2742.4 \mathrm{~m}$ depth have been investigated using the $2.2 \mathrm{MeV}$ proton beam (proton induced X-ray emission technique). Next, measurements of the porosity and the specific surface area of the pore space have been carried out using the X-ray microtomography technique. Basing on microtomographic data obtained with the high spatial resolution, simulations of the fluid dynamic in the void space of porous media have been carried out. Lattice Boltzmann method in the 3DQ19 geometrical model has been used in order to predict the hydraulic permeability of the media. In order to avoid viscositypermeability dependence the multiple-relaxation-time model with half-way bounce back boundary conditions has been used. Computing power-consuming processing has been performed with the use of modern grid infrastructure.
\end{abstract}

PACS: 81.70.Tx, 91.65. $-\mathrm{n}, 47.56 .+\mathrm{r}$

\section{Introduction}

Fluid flow in porous media is very important and common phenomenon of Nature [1]. As examples one can point a transport of water in living plants or transport of chemical fertilizer in soils. Investigations of the fluid flow in porous media have also an important significance for industrial applications (waste disposal, water purification, petroleum industry, engineering of thermal insulation materials, etc.). In this work the preliminary investigations of porous sandstone rock samples in the field of petroleum geology have been carried out. Evaluation of the porosity, specific surface area and hydraulic permeability of samples is a crucial issue for oil extraction

* corresponding author; e-mail: jakub.bielecki@ncbj.gov.pl strategy. Combination of the PIXE method, X-ray computed microtomography technique and numerical calculations for simulation of the fluid dynamics enable to obtain complex information on microstructure and chemical composition of the samples, providing the better understanding of processes important for oil research industry.

Among many nuclear techniques employed for various studies in many disciplines the proton induced X-ray emission (PIXE) method has proved to be outstanding and powerful for determination of the concentration of various elements at ppm levels and has been routinely used for study of geological samples $[2,3]$. The computed microtomography $(\mu \mathrm{CT})$ is one of the most versatile techniques for the three-dimensional examination of solid objects. The method has been already used in petro-physics for determination of microstructure prop- 
erties such as total porosity and pore size distribution [4]. Nowadays, the rapid development of computer hardware, coupled with advances in the algorithmic processing such as parallel computing or usage of powerful GPU processors enables conducting virtual experiments in the form of computer simulations on relatively large systems. Performing calculations with the use of a grid infrastructure seems to be especially promising approach as it offers huge computational resources by means of distributed computing [5].

This study describes determination of elemental composition and measurements of the particular physical properties of geological samples by means of the nuclear and X-ray microprobes at the Institute of Nuclear Physics, Polish Academy of Sciences (IFJ PAN) in Kraków. The special emphasis has been put on the $\mu \mathrm{CT}$ method. Measurements have been carried out in close cooperation with Department of Geophysics, FGGEP AGH in Kraków. Chemical composition of sandstone rock samples (few millimeters diameter), extracted from a borehole at $2679.6 \mathrm{~m}, 2741.4 \mathrm{~m}$ and $2742.4 \mathrm{~m}$ depth have been investigated using the $2.2 \mathrm{MeV}$ proton beam (PIXE technique). Next, measurements of the porosity and the specific surface area of the pore space have been carried out using the X-ray $\mu \mathrm{CT}$ technique. Basing on microtomographic data obtained with the high spatial resolution, simulations of the fluid dynamic in void space of porous media have been carried out. Lattice Boltzmann method in the 3DQ19 geometrical model has been used in order to predict the hydraulic permeability of the media. In order to avoid viscosity-permeability dependence the multiple-relaxation-time model with half-way bounce back boundary conditions has been used. Computing power-consuming processing has been performed with the use of modern grid infrastructure [6].

\section{Experimental}

\subsection{Samples description and preparation}

The objects of the investigations are samples of the Rotliegend sandstone rocks extracted from a borehole CG5 located in the region of the Foresudetic Monocline (central-western part of Poland) at $2679.6 \mathrm{~m}, 2741.4 \mathrm{~m}$ and $2742.4 \mathrm{~m}$ depth. The samples differ in respect of physical properties such as colour, brittleness, porosity, hydraulic permeability and chemical composition. Figure 1 shows a photo of the sandstone rock samples. For the PIXE measurements, samples were powdered in the

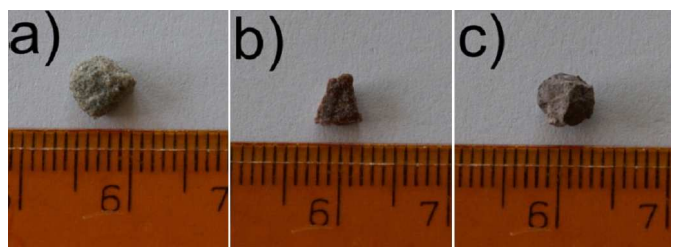

Fig. 1. Samples of the Rotliegend sandstones extracted from borehole CG5 at depth of (a) $2679.6 \mathrm{~m}$, (b) $2741.4 \mathrm{~m}$, (c) $2742.4 \mathrm{~m}$. agate mortar in order to obtain a homogeneous substance. The known amount of the powder was pressed under pressure of $15 \mathrm{MPa}$ into pellets of $10 \mathrm{~mm}$ in diameter and $0.25 \mathrm{~mm}$ thick. Each pellet was attached to the aluminum frame by a Scotch tape [7]. The $\mu \mathrm{CT}$ experiments have been carried out on about few millimeters size samples in natural form without any special sample preparation.

\subsection{PIXE analysis}

The determination of elemental concentration of trace elements has been carried out by means of the PIXE technique. A proton beam of $2.2 \mathrm{MeV}$ delivered from the Van de Graaff accelerator (HVEC K-3000) has been used. The true microprobe mode of operation was not needed, therefore the beam has been collimated down to approximately $1 \mathrm{~mm}^{2}$ on the sample. The induced $\mathrm{X}$-rays were registered by the CANBERRA Si $(\mathrm{Li})$ detector characterized by the energy resolution of $160 \mathrm{eV}$ at $5.9 \mathrm{keV}$. Aluminum absorber, $40 \mu \mathrm{m}$ thickness, has been used in order to suppress the low energy radiation. Small fraction of the proton beam, backscattered from the thin $\mathrm{Au}$ foil mounted in the ion track, was registered by a silicon surface-active detector and used for X-ray spectra normalization. The acquisition time of the characteristic $\mathrm{X}$-ray spectrum was set typically to $600 \mathrm{~s}$ for each sample, which corresponded to the charge of about $0.25 \mu \mathrm{C}$. The detector dead time was less than $10 \%$ during entire measurement. For quantitative determination of trace elements concentrations following external standards have been used: IAEA-SD-N-1/2, IAEA-SL-1, IAEA-SOIL-7 [8] and the data processing has been carried out with the use of the Guelph PIXE code WinGupix v.2.1.4 [9].

\subsection{Study of samples microstructure by means of the $\mu C T$ technique}

The $\mu \mathrm{CT}$ measurements of the porous sandstone rock samples have been carried out with the use of an experimental line of the X-ray microprobe at the IFJ PAN. Figure 2 shows a schematic view of the $\mu \mathrm{CT}$ setup. The line consists of an open type X-ray tube (Hamamatsu L9191) with microfocusing down to about $2 \mu \mathrm{m}$ [10], a high resolution X-ray sensitive CCD camera (Photonic Science $70 \mathrm{~mm}$ VHR) and a high precision rotary stage (Kohzu RA07A-W). Depending on the required X-ray energy, the $\mathrm{X}$-ray tube is used with $\mathrm{Ti}, \mathrm{Mo}, \mathrm{Ag}$, or $\mathrm{W}$ targets. In this measurement Mo target $\left(E_{K \alpha}=17.48 \mathrm{keV}\right)$ has been used.

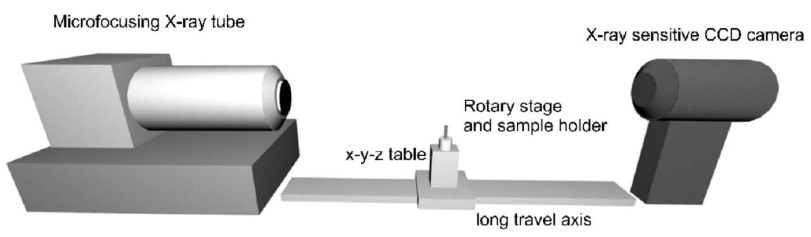

Fig. 2. Schematic view of the $\mu \mathrm{CT}$ setup. 
The tube acceleration voltage can be changed in the 20-160 kV range, whereas the tube current may be set up to maximum $200 \mu \mathrm{A}$. Radiation is emitted into a cone (120 degrees opening angle) through $0.5 \mathrm{~mm}$ thick beryllium output window. This geometry of the X-ray beam is used for tomography experiments without using any additional focusing devices. Cone beam geometry entails that projections have to be acquired with equiangular separation over $360^{\circ}$. The sensor of the CCD detector is composed of $4008 \times 2670$ pixels, readout at $10 \mathrm{MHz}$ frequency, followed by digitization of the CCD signal to 12-bit accuracy (4096 grey levels). Each pixel has size of $14.7 \mu \mathrm{m}$ in diameter. The sensor is optically bonded to a tapered fibre-optic. The fibre ending has been covered with the X-ray scintillator (gadolinium oxysulphide doped with terbium, approximately $10 \mathrm{mg} / \mathrm{cm}^{2}$ ). The thickness of the scintillator is optimized to register X-rays of energies in a range of 5 to $35 \mathrm{keV}$. The image readout is carried out by means of a commercial frame grabber card installed in a personal computer. Rotating of a sample during $\mu \mathrm{CT}$ measurement is accomplished with the use of rotary stage with repeatability of about $0.03^{\circ}$. The stage is equipped with Reinshaw encoder working with feedback mode in order to precise control of the stage position. The rotary stage has been mounted on manual $x-y-z$ table allowing alignment of the sample rotation axis. A small focus size and short focus-to-object distance enable to obtain images of samples with high magnification and the resolution of the order of few micrometers. The $\mu \mathrm{CT}$ measurements are carried out using home developed codes combined with commercial CT reconstruction software [11].

\subsection{Media permeability assessment by means of lattice Boltzmann method}

Lattice Boltzmann method (LBM) is a class of computational fluid dynamics (CFD) techniques for simulation of fluid flow. It is a relatively new, but proven to be reliable $[12,13]$ simulation technique for complex fluid systems, which has attracted interest of computational physics researchers. In the LBM instead of solving the Navier-Stokes equations, the discrete Boltzmann equation is solved in order to simulate the flow of a Newtonian fluid with collision models such as BhatnagarGross-Krook (BGK) [14]. Unlike other CFD methods, which solve the conservation equations of the macroscopic properties i.e. mass, momentum, and energy numerically, LBM models the fluid consisting of fictive particles, which perform consecutive propagation and collision processes over a discrete lattice mesh. In this computations the D3Q19 (3 dimensions, 19 discrete velocity vectors) model has been used (Fig. 3). Due to its particulate nature and local dynamics, LBM has several advantages over other conventional CFD methods, especially in dealing with complex boundaries which occur in porous media. Due to the locality of the collision operator LBM is highly suitable for parallel calculations which justifies usage of a distributed computational resources. In order to avoid viscosity-permeability dependence the multiple-relaxation-time (MRT) model with half-way bounce back boundary conditions has been used [15].

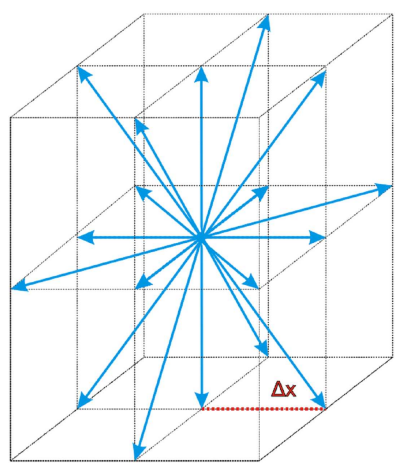

Fig. 3. Illustration of 19 discrete velocity vectors available in the D3Q19 model used in simulations.

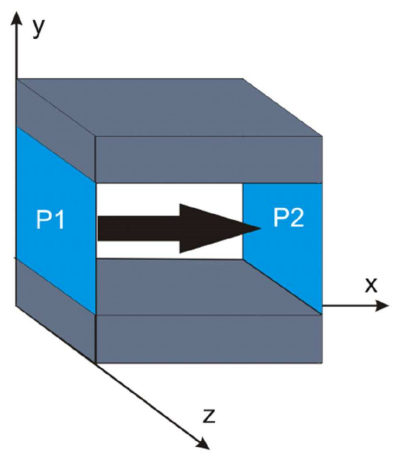

Fig. 4. Geometry of the Poiseuille flow used in a benchmark simulation. Velocity profile in the $y$ direction is parabolic.

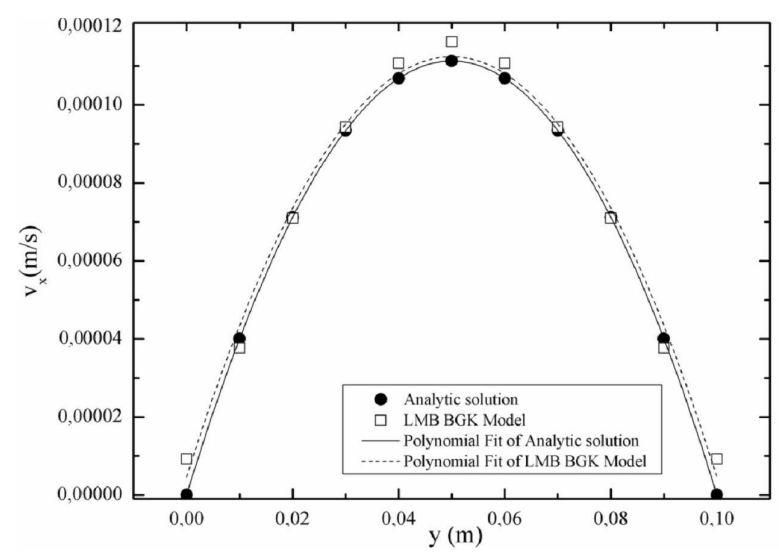

Fig. 5. Fluid $x$ velocity component as a function of $y$ coordinate. Simulation results vs. analytical solution.

For all LBM simulations an open-source LBM solver Palabos [16] has been used. In order to check the correctness and performance of the used code a test run simulat- 
ing Poiseuille flow between two parallel plates (Fig. 4) has been carried out. The vertical velocity profile has been obtained and compared with analytical solution (Fig. 5). A good agreement of simulation with analytical prediction was observed.

The permeability $k$ of the medium was determined using the Darcy law

$$
\left\langle q_{x}\right\rangle=\frac{k}{\mu} \frac{\mathrm{d} P}{\mathrm{~d} x},
$$

where $P$ - pressure, $\left\langle q_{x}\right\rangle$ - volumetric average of fluid flux, $\mu$ - dynamic viscosity. Simulations were performed on $255 \times 255 \times 255$ lattice mesh (voxel physical dimensions $20 \times 20 \times 20 \mu \mathrm{m}^{3}$ ) with geometry of the medium obtained from $\mu \mathrm{CT}$ measurements and binarized into two regions — fluid and void space.

\section{Results}

The X-ray spectra processing by the Gupix package has been done assuming that the samples matrix is composed entirely from $\mathrm{SiO}_{2}$. Chi-square value of the fits varied from 2.1 to 10.7. All PIXE results obtained in this study are summarized in Table I.

TABLE I

The PIXE results of elemental concentration in sandstones samples. $<$ MDL means that calculated concentration was below minimum detection limit.

\begin{tabular}{|c|c|c|c|c|c|c|}
\hline \multirow{2}{*}{$\begin{array}{c}\text { Depth } \\
{[\mathrm{m}]}\end{array}$} & \multicolumn{2}{|c|}{2679.6} & \multicolumn{2}{|c|}{2741.4} & \multicolumn{2}{|c|}{2742.4} \\
\hline & [ppm] & $\pm[\mathrm{ppm}]$ & [ppm] & $\pm[\mathrm{ppm}]$ & [ppm] & $\pm[\mathrm{ppm}]$ \\
\hline $\mathrm{Al}$ & 180 & 20 & 370 & 30 & 60 & 5 \\
\hline $\mathrm{P}$ & \multicolumn{2}{|c|}{$<\mathrm{MDL}$} & 350 & 20 & \multicolumn{2}{|c|}{$<\mathrm{MDL}$} \\
\hline S & 320 & 10 & 440 & 20 & \multicolumn{2}{|c|}{$<\mathrm{MDL}$} \\
\hline $\mathrm{Cl}$ & 5000 & 50 & 3600 & 40 & 6600 & 40 \\
\hline K & 20000 & 100 & 9800 & 65 & 6000 & 30 \\
\hline $\mathrm{Ca}$ & 15500 & 200 & 2000 & 35 & 3200 & 20 \\
\hline $\mathrm{Ti}$ & 3200 & 45 & 3000 & 40 & 740 & 10 \\
\hline $\mathrm{V}$ & \multicolumn{2}{|c|}{$<\mathrm{MDL}$} & \multicolumn{2}{|c|}{$<\mathrm{MDL}$} & 80 & 10 \\
\hline $\mathrm{Mn}$ & 350 & 50 & \multicolumn{2}{|c|}{$<\mathrm{MDL}$} & 320 & 15 \\
\hline $\mathrm{Fe}$ & 30000 & 200 & 11000 & 100 & 28700 & 100 \\
\hline $\mathrm{Cu}$ & 230 & 20 & 260 & 20 & 120 & 20 \\
\hline
\end{tabular}

The concentrations of most of the determined trace elements are in reasonable agreement with concentrations of elements in sandstones rocks reported in other works $[2,17,18]$, however higher concentration level of $\mathrm{Cl}$ has been observed in current study due to presence of brine as a formation water in porous space. Concentrations of $\mathrm{Zn}, \mathrm{Ga}, \mathrm{Rb}, \mathrm{Sr}$ and $\mathrm{W}$ as well as, in some cases, of $\mathrm{P}$, $\mathrm{S}, \mathrm{V}$, and $\mathrm{Mn}$ were below minimum detection limit.

The porosity, specific surface area and pore size distribution have been determined with the use of home-developed code basing on obtained $\mu \mathrm{CT}$ images. An example of reconstructed three-dimensional microtomographic image of the sandstone rock is shown in Fig. 6. The average porosity of the sample from $2679.6 \mathrm{~m}$ and $2741.4 \mathrm{~m}$ depth has been evaluated as $18.7 \pm 3.4 \%$ (Fig. 7 ) and $11.1 \pm 3.4 \%$ (Fig. 8), respectively. The results of the porosity measurement are in reasonable agreement with results obtained with the use of the mercury porosimetry technique (15.28\% for the sample from $2679.6 \mathrm{~m}$ depth). Pore size distributions for the above mentioned samples are shown in Fig. 9 and Fig. 10. Table II presents results of the specific surface area (a property of solids which measures the total surface area of porous media per unit of volume) measurement.

\section{TABLE II}

Results of the specific surface area evaluation.

\begin{tabular}{c|c|c}
\hline \hline \multirow{2}{*}{$\begin{array}{c}\text { Depth } \\
{[\mathrm{m}]}\end{array}$} & \multicolumn{2}{|c}{ Specific surface area } \\
\cline { 2 - 3 }$\left[\mu \mathrm{m}^{-1}\right]$ & $\pm\left[\mu \mathrm{m}^{-1}\right]$ \\
\hline 2679.6 & 0.0266 & 0.0012 \\
2741.4 & 0.0294 & 0.0013
\end{tabular}

An open source LBM solver "Palabos" [16] has been used in order to calculate total permeability of the media. The average permeability of the sample from $2679.6 \mathrm{~m}$ depth has been estimated basing on the Darcy law to $114.0 \pm 2.8 \mathrm{mD}\left(1 \mathrm{D}=9.869233 \times 10^{-13} \mathrm{~m}^{2}\right)$, whereas the average permeability of the sample from $2741.4 \mathrm{~m}$ is 


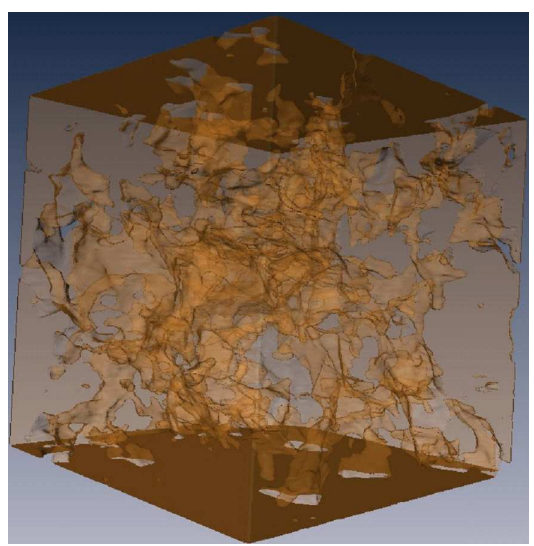

Fig. 6. An example of reconstructed three-dimensional microtomographic image of the sandstone rock.

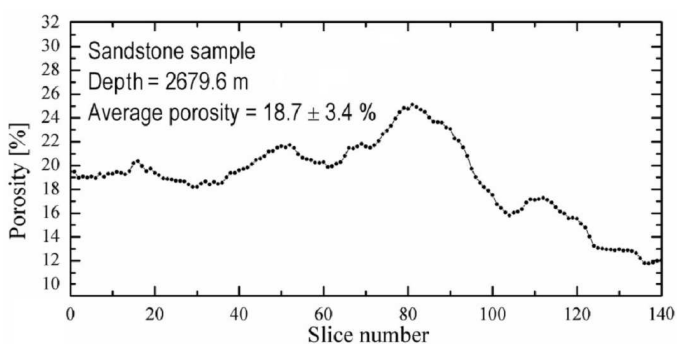

Fig. 7. Results of the porosity assessment of the sandstone sample from $2679.6 \mathrm{~m}$.

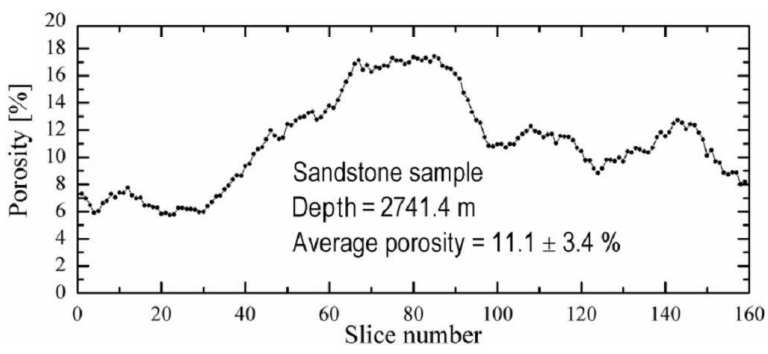

Fig. 8. Results of the porosity assessment of the sandstone sample from depth of $2741.4 \mathrm{~m}$.

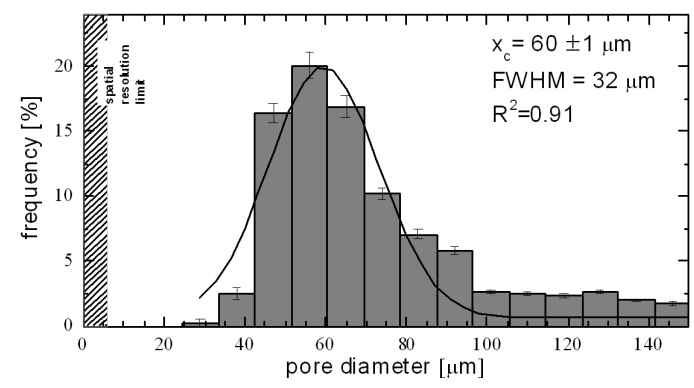

Fig. 9. Pore size distribution in the sample from $2679.6 \mathrm{~m}$ depth.

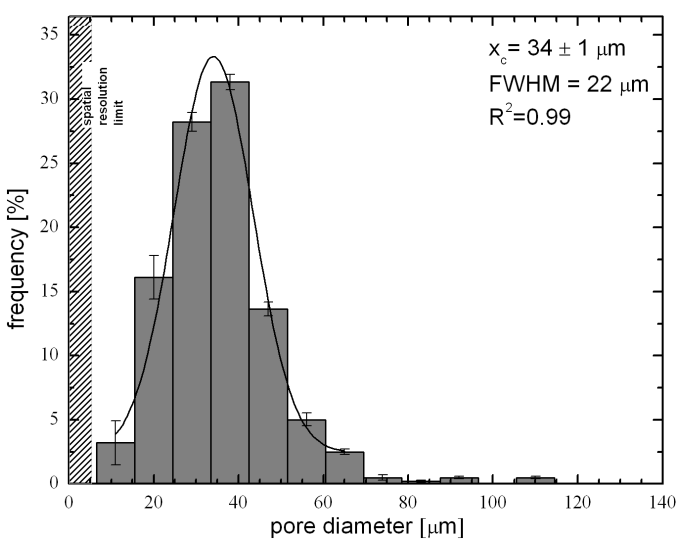

Fig. 10. Pore size distribution in the sample from $2741.4 \mathrm{~m}$ depth.

$0.49 \pm 0.02 \mathrm{mD}$. The obtained values are within the range of the oil reservoir rocks permeability.

\section{Conclusion}

In this work two experimental methods combined with numerical simulations have been used in order to study elemental content, microstructure and physical properties of the geological porous media. Two facilities: nuclear microprobe and the $\mu \mathrm{CT}$ experimental line of X-ray microprobe have been used for this purpose. Presented investigations are preliminary, however obtained results are in reasonable agreement with results obtained with the use of other techniques and results reported by others researchers. The research will be continued and home-made software for porous media analysis used in these studies is still under development.

\section{Acknowledgments}

The authors would like to thankfully acknowledge: Ministry of Science and Higher Education of the Republic of Poland (grant no. NN 507 298339), Polish Infrastructure for Information Science Support in the European Research Space PL-Grid Project (access to the computational grid infrastructure), support in the form of the DOCTUS scholarship cofounded by European Social Fund, MP0601 COST Action — "Short Wavelength Laboratory Sources".

\section{References}

[1] P. Wong, Methods of the Physics of Porous Media, Experimental Methods in the Physical Sciences, Vol. 35, Academic Press, San Diego (CA) 1999.

[2] S. Olabanji, A. Haque, S. Zandolin, R. Ajayi, M. Buoso, D. Ceccato, R. Cherubini, D. Zafiropolos, G. Moschini, Nucl. Instrum. Methods Phys. Res. B 109/110, 262 (1996). 
[3] P. Sonck-Koota, A. Lindroos, J. Lill, J. Rajander, E. Viitanen, F. Marra, M. Pehkonen, J. Suksi, S. Heselius, Nucl. Instrum. Methods Phys. Res. B 266, 2367 (2008).

[4] C. Appoloni, C. Fernandes, C. Rodrigues, Nucl. Instrum. Methods Phys. Res. A 580, 629 (2007).

[5] J. Harting, J. Chin, M. Venturoli, P. Coveney, Philos. Trans. R. Soc. A 363, 1895 (2005).

[6] http://www.plgrid.pl/ .

[7] G. Ghermandi, R. Cecchi, P. Laj, Nucl. Instrum. Methods Phys. Res. B 109/110, 63 (1996).

[8] http://curem.iaea.org/catalogue/TE/ .

[9] http://pixe.physics.uoguelph.ca/gupix/main/ .

[10] J. Bielecki, S. Bożek, J. Lekki, Z. Stachura, W.M. Kwiatek, Acta Phys. Pol. A 115, 537 (2009).

[11] http://www.inct.be/en/home .
[12] C. Aidun, J. Clausen, Ann. Rev. Fluid Mech. 42, 439 (2010).

[13] S. Chen, G. Doolen, Ann. Rev. Fluid Mech. 30, 329 (1998).

[14] P. Bhatnagar, E. Gross, M. Krook, Phys. Rev. 94, 511 (1954).

[15] C. Pan, L. Luo, C. Miller, Comput. Fluids 35, 898 (2006).

[16] http://www.lbmethod.org/palabos/.

[17] H. Mahmoud, A. Abbady, M. Khairy, A. Abdehalim, A. El-Taher, J. Radioanal. Nucl. Chem. 264, 715 (2005).

[18] C. Rezende, L. Lacerda, A. Ovalle, C. Souza, A. Gobo, D. Santos, Mar. Pollut. Bull. 44, 680 (2002). 